\title{
Bio-sensing applications of graphene based composite films
}

\begin{abstract}
Graphene based composite materials have been extensively studied for the sensing applications attributing to their 2D structures, high conductivity, controlled modification and large specific surface areas, unique mechanical, optical, chemical, electrical, and catalytic properties. Therefore, a number of high quality sensors have been fabricated in recent years. Graphene based composite films (GCFs) that is base of such sensors can be prepared by combining Graphene with different functional nano-materials (carbon materials, noble metals, polymer materials, metal compounds etc.). In this review, we focus on the recent advances in bio-sensing applications of Graphene based composite films.
\end{abstract}

Volume 3 Issue 2 - 2017

Savita Maurya

Dept of Electronics \& Communication Engineering, Integral

University, India

Correspondence: Savita Maurya, Dept of Electronics \& Communication Engineering, Integral University, Kurshi Road, Lucknow-226026, India, Email mauryasavita5@gmail.com

Received: July 27, 2017 | Published: September 22, 2017

Keywords: Graphene, GCFs, 2D, RGO, QD, bio-sensor

\section{Introduction}

Graphene is a two-dimensional (2D) material having honey-comb crystal lattice and thickness of one-atom. It has unique mechanical, electronic, chemical, optical, and thermal properties..$^{1-5}$ Particularly its one atom thickness, high charge mobility and high surface-to-volume ratio make it eligible for very sensitive sensing applications. ${ }^{6-7}$ Applicability of GCFs for various applications is also limited by their fabrication process. Graphene based biological sensors fabricated through screen-printing electrochemical process has some pros and cons. ${ }^{8}$ In this review, we focus on the recent advances in bio-sensing application of Graphene based composite films. Bio sensors are discussed based on usage and sensing mechanism. The key difficulties and future points of view in this quickly emerging field going for GCFs for future sensing applications are given.

\section{Discussion}

Various biologically- relevant substances/biomaterials such as DNA, blood sugar, other parameters and $\mathrm{H}_{2} \mathrm{O}_{2}$ can be detected using Graphene and/ or its composite films. ${ }^{9-15}$ We will discuss GCF based bio-sensors according to their sensing mechanism.

\section{Photo-electrochemical (PEC) bio-sensor}

Photo catalytic oxidation/reduction of molecules produces improved electron transfer between semiconductor and analyte when light falls on it. This is the basic principle of Photoelectro-chemical bio-sensors. Generally, quantum dots are used as visible-light active materials. Authors in ${ }^{16}$ used CdS QDs-DNA-Graphene composite film as modified electrode. Very high conductivity of Graphene has improved the photo- current significantly. This highly sensitive and high stability PEC sensor can be used to track genotoxic pollutants.

\section{Field-effect transistor (FET) based bio-sensor}

Conduction of channel region of FET changes upon adsorption of target molecules, and this is the basis of FET based bio-sensors. Authors in ${ }^{17}$ discussed FET based biosensor to detect cholesterol. Authors in ${ }^{18}$ developed a cholesterol sensor using NiO-Graphene nano composite film. Authors in ${ }^{19}$ developed DNA FET sensor using AuNPs-Graphene composite films.

\section{Enzymatic \& non-enzymatic electro chemical biosensor}

Authors in ${ }^{20}$ fabricated a new electrochemical sensor for sensing application of $\mathrm{H}_{2} \mathrm{O}_{2}$. Non-enzymatic sensors have some advantages over their enzymatic counterpart such as cheap fabrication, reusability, wide detection range, excellent selectivity and high sensitivity. Authors in ${ }^{21}$ fabricated $\mathrm{MnO}_{2}$ - RGO (Reduced Graphene Oxide) film modified electrode based biosensor. Authors in ${ }^{22}$ also reported RGOPLL- $\mathrm{Mn}_{3} \mathrm{O}_{4}$ based biosensor with improved catalytic activity toward glucose. Authors in $^{23,24}$ have reported, non enzymatic $\mathrm{H}_{2} \mathrm{O}_{2}$ sensor based on RGO-AuNP hybrid membranes.

\section{Fluorescent biosensor}

Fluorescent bio-sensors are based on energy transfer due to fluorescence resonance, and simultaneous multiplex target detection. Authors in ${ }^{25}$ developed Graphene quantum dots (GQDs) based biosensor to detect hydrogen peroxide $\left(\mathrm{H}_{2} \mathrm{O}_{2}\right)$ and glucose in diabetes patients. This same sensor can be used as electro-chemical and fluorescent bio-sensor. Authors in $^{14}$ also reported GO-AuNPs fluorescence system for DNA sensing.

\section{Other Graphene biosensors}

Proteins have charges/dipoles those changes under physiological conditions and this made them suitable for electronic detection of proteins using scattering of field effect. ${ }^{9}$ Authors in $^{26}$ reported sensor for bacteria detection with very high sensitivity (up to single-cell level). Highly sensitive Graphene hybrid nano-sensors can be directly integrated with biomaterials. These are battery- free that can be used for remote monitoring of pathogenic bacteria and food safety analysis. $\mathrm{WS}_{2} / \mathrm{Au}$ NPs based bio interfaces were fabricated for $17 \mathrm{~b}$-estradiol. ${ }^{27}$ Authors in ${ }^{28}$ reported, complex MWCNT/MoS $/ \mathrm{Au} / \mathrm{GOx}$ based bio interfaces for DNA sensing. Table 1 summarizes different biosensors based on GCFs. 
Table I Biosensors based on GCFs.

\begin{tabular}{lll}
\hline Materials & Analyte & References \\
\hline RGO-ZnO & DNA \& TNT & 13 \\
G-CdS-DNA & Catechol & 16 \\
G-NiO & Cholesterol & 18 \\
G-AuNPs & DNA & 19 \\
RGO-AuNP & $\mathrm{H}_{2} \mathrm{O}_{2}$ & 20 \\
RGO-PLL-MnO & ${ }_{2}$ & ${ }^{21}$ \\
RGO-PLL-Mn ${ }_{3} \mathrm{O}_{4}$ & $\mathrm{Glucose}$ oxidase, $\mathrm{H}_{2} \mathrm{O}_{2}$ & 21 \\
RGO-AuNPs non-enzymatic & $\mathrm{H}_{2} \mathrm{O}_{2}$ & 22 \\
RGO-AuNPs- non-enzymatic & $\mathrm{H}_{2} \mathrm{O}_{2}$ & 23 \\
WS $/$ Au NPs & I7b-estradiol & 24 \\
MWCNT/MoS /Au/GOx & DNA & 27 \\
Aminophenylboronic acid & Glucose, glycated & 29 \\
(APBA)-functionalized RGO & hemoglobin & 28 \\
Thrombin binding aptamer & thrombin & \\
(TBA)/GOx & & 20
\end{tabular}

\section{Conclusion}

Two dimensional Graphene, GFCs and Graphene-like nanomaterials is a class of emerging nano-materials with specific planar morphology and properties have attracted considerable attentions. Taking the advantage of chemical compositions and assorted biological effects, these possess attractive and matchless properties and provide colossal opportunity for their ample applications. This review article highlights the recent progress in the development of GFC based bio-interfaces for their bio-sensing applications. Some researchers have done in-depth analysis and reported that there are plenty of possibilities to prepare different GFC based biosensors as more reactions and more molecular structure changes can be taken as bridged media of biosensors. Availability of large surface-to-volume ratio and recognition ability of the biological molecules reactions of Graphene materials increases the selectivity and sensitivity of the biosensors. But everything is not ok with GFC based sensors high salt concentration is one of them. High salt concentration changes surface charge arrangement of GFCs by its aggregation and precipitation. ${ }^{29,30}$ It is clear that the immense possibilities in terms of synthesizing Graphene and GFCs and fabricating functional bio-interfaces will lead to the fast development in this hot research area.

\section{Acknowledgements}

None.

\section{Conflict of interest}

The author declares no conflict of interest.

\section{References}

1. He Q, Wu S, Gao S, et al. Transparent, flexible, all-reduced graphene oxide thin film transistors. ACS Nano. 2011;5(6):5038-5044.

2. Liu G, Jin W, Xu N. Electronic structures and theoretical modelling of two-dimensional group-VIB transition metal dichalcogenides. Chem Soc Rev. 2015;44:5016-5030.
3. Lee JH, Lee EK, Joo WJ, et al. Wafer-scale growth of single-crystal monolayer graphene on reusable hydrogen-terminated germanium. Science. 2014;344(6181):286-289.

4. Pumera M. Graphene-based nanomaterials and their electrochemistry. Chem Soc Rev. 2010;39(11):4146-4157.

5. Yang Z, Ren J, Zhang Z, et al. Recent advancement of nanostructured carbon for energy applications. Chem Rev. 2015;115(11):5159-5223.

6. Ding J, Sun W, Wei G, et al. Cuprous oxide microspheres on graphene nanosheets: an enhanced material for non-enzymatic electrochemical detection of $\mathrm{H} 2 \mathrm{O} 2$ and glucose. RSC Adv. 2015;5:35338-35345.

7. Zhang P, Lu X, Huang Y, et al. J Mater Chem. 2015. p. 14562-14566.

8. Cinti P, Arduini F. Graphene-based screen-printed electrochemical (bio) sensors and their applications: Efforts and criticisms. Biosensors and Bioelectronic. 2017;89(1):107-122.

9. Salavagione HJ, Díez-Pascual AM, Lázaro E, et al. J Mater Chem A2. 2014. p. 14289-14328.

10. Turcheniuk K, Boukherroub R, Szunerits $\mathrm{S}$. Gold-graphene nanocomposites for sensing and biomedical applications. J Mater Chem B3. 2015. p. 4301-4324.

11. Wang J, Ouyang Z, Ren Z, et al. Carbon. 2015;89:20-30.

12. Wang J, Rathi S, Singh B, et al. Alternating Current dielectrophoresis optimization of pt-decorated graphene oxide nanostructures for proficient hydrogen gas sensor. ACS Appl Mater Interfaces. 2105;7(25):1376813775 .

13. Yang T, Chen M, Kong Q, et al. Shape-controllable $\mathrm{ZnO}$ nanostructures based on synchronously electrochemically reduced graphene oxide and their morphology-dependent electrochemical performance. Electrochim Acta. 2015; 182:1037-1045.

14. Liu Y, Dong X, Chen P. Biological and chemical sensors based on graphene materials. Chem Soc Rev. 2012;41:2283-2307.

15. Zhang M, Li Y, Su Z, et al. Polym Chem. 2015;6:6107-6124.

16. Liu Y, Wang R, Zhu Y, et al. A facile label-free colorimetric method for highly sensitive glutathione detection by using manganese dioxide nanosheets. Sens Actuators B. 2015;242:355-361.

17. He Q, Wu S, Yin Z, et al. Graphene-based electronic sensors. Chem Sci. 2013;6:1764-1772.

18. Rengaraj A, Haldorai Y, Kwak CH, et al. Electrodeposition of flowerlike nickel oxide on CVD-grown graphene to develop an electrochemical non-enzymatic biosensor. J Mater Chem . 2015;3:6301-6309.

19. Peng HP, Hu Y, Liu P, et al. Label-free electrochemical DNA biosensor for rapid detection of mutidrug resistance gene based on Au nanoparticles/ toluidine blue-graphene oxide nanocomposites. Sens Actuators. 2015;B207:269-276.

20. Xi Q, Chen X, Evans DG, et al. Gold nanoparticle-embedded porous graphene thin films fabricated via layer-by-layer self-assembly and subsequent thermal annealing for electrochemical sensing. Langmuir. 2012;28:9885-9892.

21. Vilian ATE, Mani V, Chen SM, et al. Ind Eng Chem Res. 2014;53:558215589 .

22. Yang S, Li G, Wang G, et al. Synthesis of $\mathrm{Mn}_{3} \mathrm{O}_{4}$ nanoparticles/nitrogendoped graphene hybrid composite for nonenzymatic glucose sensor. Sens Actuators. 2015;B221:172-178.

23. Zhang $\mathrm{P}$, Huang $\mathrm{Y}$, Lu $\mathrm{X}$, et al. One-step synthesis of large-scale graphene film doped with gold nanoparticles at liquid-air interface for electrochemistry and Raman detection applications. Langmuir. 2013;30(29):8980-8989. 
24. Zhang P, Zhao X, Zhang S, et al. J Mater Chem B1. 2013. p. 6525-6531.

25. Zhang P, Zhao X, Ji Y, et al. J Mater Chem B3. 2015. p. 2487-2496.

26. Mannoor MS, Tao H, Clayton JD, et al. Graphene-based wireless bacteria detection on tooth enamel. Nat Commun. 2012;3:763.

27. Huang KJ, Liu YJ, Wang HB, et al. Sub-femtomolar DNA detection based on layered molybdenum disulfide/multi-walled carbon nanotube composites, Au nanoparticle and enzyme multiple signal amplification. Biosens Bioelectron. 2014:55:195-202.
28. Huang KJ, Liu YJ, Zhang JZ, et al. A novel aptamer sensor based on layered tungsten disulfide nanosheets and Au nanoparticles amplification for 17ß-estradiol detection. Anal Methods. 2014;6:8011-8017.

29. Sridevi S, Vasu KS, Sampath S, et al. Optical detection of glucose and glycated hemoglobin using etched fiber Bragg gratings coated with functionalized reduced graphene oxide. J Biophotonics. 2016;9(7):760 769

30. Ahour F, Ahsani MK. An electrochemical label-free and sensitive thrombin aptasensor based on graphene oxide modified pencil graphite electrode. Biosens Bioelectron. 2016;86:764-769. 